\title{
Fluorescence Detection of Cell Death in Liver of Mice Treated with Thioacetamide
}

\author{
Jin Seok Kang ${ }^{1,2}$ \\ ${ }^{1}$ Department of Biomedical Laboratory Science, Namseoul University, Cheonan, Korea \\ ${ }^{2}$ Molecular Diagnostics Research Institute, Namseoul University, Cheonan, Korea
}

\begin{abstract}
The purpose of this study was to detect cell death in the liver of mice treated with thioacetamide (TAA) using fluorescence bioimaging and compare this outcome with that using conventional histopathological examination. At 6 weeks of age, 24 mice were randomly divided into three groups: group 1 (G1), control group; group 2 (G2), fluorescence probe control group; group 3 (G3), TAA-treated group. G3 mice were treated with TAA. Twenty-two hours after TAA treatment, G2 and G3 mice were treated with Annexin-Vivo 750. Fluorescence in vivo bioimaging was performed by fluorescence molecular tomography at two hours after Annexin-Vivo 750 treatment, and fluorescence ex vivo bioimaging of the liver was performed. Liver damage was validated by histopathological examination. In vivo bioimaging showed that the fluorescence intensity was increased in the right upper part of G3 mice compared with that in G2 mice, whereas G1 mice showed no signal. Additionally ex vivo bioimaging showed that the fluorescence intensity was significantly increased in the livers of G3 mice compared with those in $\mathrm{G} 1$ or $\mathrm{G} 2$ mice $(p<0.05)$. Histopathological examination of the liver showed no cell death in G1 and G2 mice. However, in G3 mice, there was destruction of hepatocytes and increased cell death. Terminal deoxynucleotidyl transferase dUTP nick end labeling staining confirmed many cell death features in the liver of G3 mice, whereas no pathological findings were observed in the liver of G1 and G2 mice. Taken together, fluorescence bioimaging in this study showed the detection of cell death and made it possible to quantify the level of cell death in male mice. The outcome was correlated with conventional biomedical examination. As it was difficult to differentiate histological location by fluorescent bioimaging, it is necessary to develop specific fluorescent dyes for monitoring hepatic disease progression and to exploit new bioimaging techniques without dye-labeling.
\end{abstract}

Key words: Mice, Thioacetamide, Cell death, Fluorescence bioimaging, Histopathology

\section{INTRODUCTION}

Exposure to hazardous chemicals induces toxic responses, including cell death, oxidative stress, inflammation and DNA damage. Different types of cell death occur in the body, such as necrosis and apoptosis (1). A major change in dying cells is the translocation of phosphatidylserine

Correspondence to: Jin Seok Kang, Department of Biomedical Laboratory Science, Namseoul University, 91 Daehak-ro, Seonghwan-eup, Sebuk-gu, Cheonan 31020, Korea

E-mail: kang@nsu.ac.kr

This is an Open-Access article distributed under the terms of the Creative Commons Attribution Non-Commercial License (http:// creativecommons.org/licenses/by-nc/3.0) which permits unrestricted non-commercial use, distribution, and reproduction in any medium, provided the original work is properly cited. from the inner to the outer leaflet of the plasma membrane (2), which is an attractive target for bioimaging (3).

Accurate assessment of cell death is important to investigate disease progression (4). For the conventional detection of cell death, tissues are processed for histopathological studies and general morphological examination by hematoxylin and eosin (HE) staining or specific detection of cell death by immunohistochemical staining. These conventional toxicity tests take more time to identify toxicities.

Because many chemicals and pharmaceutics have been developed, there has been increasing demands for new technologies to evaluate them more quickly and accurately. Recent advancement in bioimaging technologies has enabled the detection of specific molecules (5) and visualization of biological processes during disease progression $(6,7)$, providing quantitative results $(8)$. 
As a bioimaging technology, optical bioimaging has been used to investigate altered biological processes in vivo (9) and to detect specific molecule(s) in living condition of animals (10). Many fluorescence bioimaging agents have been developed to detect several pathological changes such as tumor cell death (11) and oxidative stress (12).

Thioacetamide (TAA) is known to be a hepatotoxicant. Short-term treatment in rodents induces acute liver cell death $(13,14)$ and long-term treatment results in fibrosis and/or cirrhosis (15), hepatic regenerative nodules (16), and hepatic tumors (17), which are associated with the activation of hepatic stellate cells (18).

To date, no report exists concerning the fluorescence detection of TAA-induced cell death using fluorescence bioimaging including control and probe control groups. Thus, this study aimed to test whether fluorescence bioimaging using near-infrared (NIR) agents would provide a real readout of cell death in mice, and compared the outcome with that using conventional histopathological examination.

\section{MATERIALS AND METHODS}

Animals and treatment. Male ICR mice (5 weeks old) were obtained from Nara Biotech Co. Ltd. (Seoul, Korea) and were acclimated for 7 days before the initiation of the study. They were kept in a temperaturecontrolled environment $\left(22 \pm 3^{\circ} \mathrm{C}\right)$ with $55 \pm 5 \%$ relative humidity and a 12-hr light/dark cycle. The mice were fed unrefined chlorophyll-containing ingredients, in particular, an alfalfa-free diet (Harlan Laboratories, Inc., Madison, WI, USA) and filtered water ad libitum. This study (NSU-15-2) was approved by the animal experiment committee of Namseoul University based on the Animal Protection Act.

At 6 weeks of age, 24 mice were randomly divided into three groups: group $1(\mathrm{G} 1 ; \mathrm{n}=8)$, control group; group 2 $(\mathrm{G} 2 ; \mathrm{n}=8)$, fluorescence probe control group; group 3 $(\mathrm{G} 3 ; \mathrm{n}=8)$, TAA-treated group. G3 mice were treated with TAA $(200 \mathrm{mg} / \mathrm{kg}$ of body weight, single, intraperitoneally) at 6 weeks of age, while G1 and G2 mice received $0.9 \%$ saline in equal volumes at equivalent time. Twentytwo hours after TAA treatment, G2 and G3 mice were treated with Annexin-Vivo 750 (PerkinElmer, Waltham, MA, USA; $100 \mu \mathrm{L} /$ mouse, intravenously) according to manufacturer's instruction. As controls, G1 mice received equal volumes of $0.9 \%$ saline at the same time. All the hair was removed from the mice by shaving and depilation with a cosmetic hair removal lotion (Veet cream; Reckitt Benckiser, Berkshire, UK) before fluorescence bioimaging analysis.

Fluorescence in vivo bioimaging. Before in vivo imaging, the mice were anesthetized with avertin (2.2.2tribromoethanol; Sigma, Saint Louis, MO, USA) at twenty- four hours after TAA treatment. Additionally, the skin and peritoneum of mice were removed, and mice were placed in fluorescence molecular tomography (FMT) 4000 (PerkinElmer) system imaging chamber and fluorescence bioimaging was conducted. Fluorescence patterns were captured with a thermoelectrically cooled CCD camera, and the position and intensity of fluorescence sources were recorded.

Fluorescence ex vivo bioimaging. Following in vivo imaging, mice were sacrificed, and the livers were excised. And bioimaging was performed immediately using fluorescence-labeled organism bioimaging instrument (FOBI) system (Neoscience, Suwon, Korea). The exposure time was $2 \mathrm{~s}$ per image, and the images were analyzed using the dedicated software.

Histopathological examination. Liver tissues were fixed in $10 \%$ neutral phosphate-buffered formalin, processed and embedded in paraffin, and $4 \mathrm{~mm}$ sections were stained with HE for histopathological examination.

Terminal deoxynucleotide transferase dUTP nick end labeling (TUNEL) staining of apoptosis. To confirm cell death in the liver, immunohistochemical analysis was carried out. The avidin-biotin complex method was used to detect cell death in 5 - $\mu \mathrm{m}$ sections of liver tissue that had been dewaxed with xylene and hydrated using a graded ethanol series. The sections were treated sequentially with proteinase $\mathrm{K}$ and $3 \%$ hydrogen peroxide, and then were treated with equilibration buffer, TdT enzyme and anti-digoxigenin peroxidase conjugate according to the manufacturer's instructions (ApopTag ${ }^{\circledR}$ Peroxidase In Situ Apoptosis Detection Kit; Chemicon International, Temecula, CA, USA). Immune complexes were visualized using DAB (Sigma) as the chromogen. Phosphatebuffered saline was used instead of the TdT enzyme, as a negative control. Sections were counterstained with hematoxylin to facilitate their examination under a light microscope.

Statistical analysis. Statistical analyses were performed using GraphPad Prism 6 (GraphPad Software, La Jolla, CA, USA). All data were analyzed using Dunnett's multiple comparison test following one-way analysis of variance and Student's $t$-test. The $p$-values $<0.05$ were considered statistically significant.

\section{RESULTS}

Fluorescence in vivo bioimaging. The mice were given intravenous injections of Annexin-Vivo 750 and then were placed horizontally and imaged sequentially by a FMT 4000 imaging system. Fluorescence intensity was 
increased in the right upper part of G3 mice compared with G2 mice, whereas G1 mice showed no signal (Fig. 1). In other parts, fluorescence intensity was not observed.
Fluorescence ex vivo bioimaging. On ex vivo optical images, high fluorescent activity of Annexin-Vivo 750 was detected in the livers of G3 mice compared with those
(A)

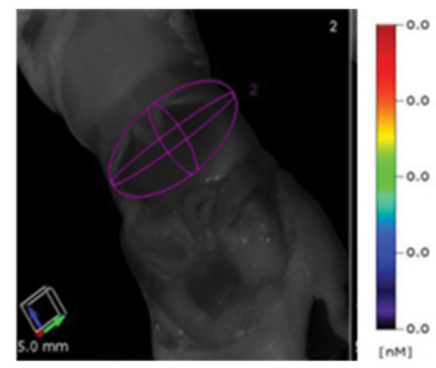

(B)

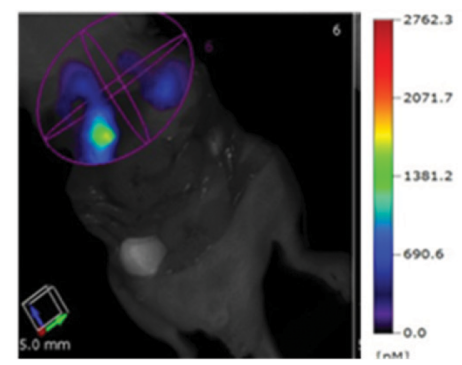

(C)

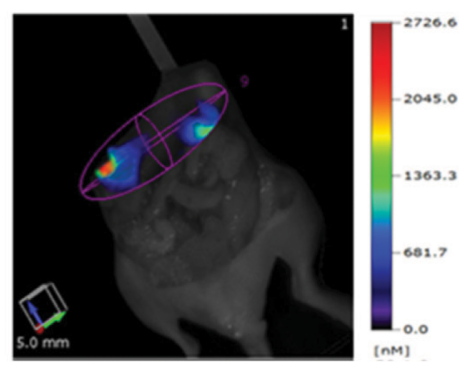

Fig. 1. Fluorescence in vivo imaging of mice detected by Fluorescence Molecular Tomography (FMT) using Annexin-Vivo 750. (A) FMT imaging of G1 as control. (B) FMT imaging of G2 as probe-treated. (C) FMT imaging of G3 as probe and thioacetamide treated. Note the high intensity level of fluorescence in right upper part of abdomen in G3.

(A)

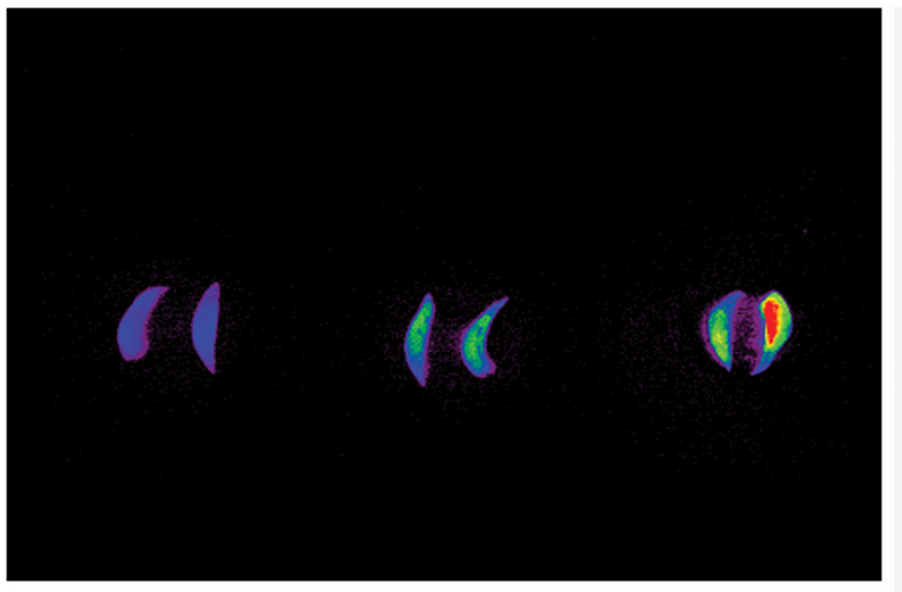

G1

(B)

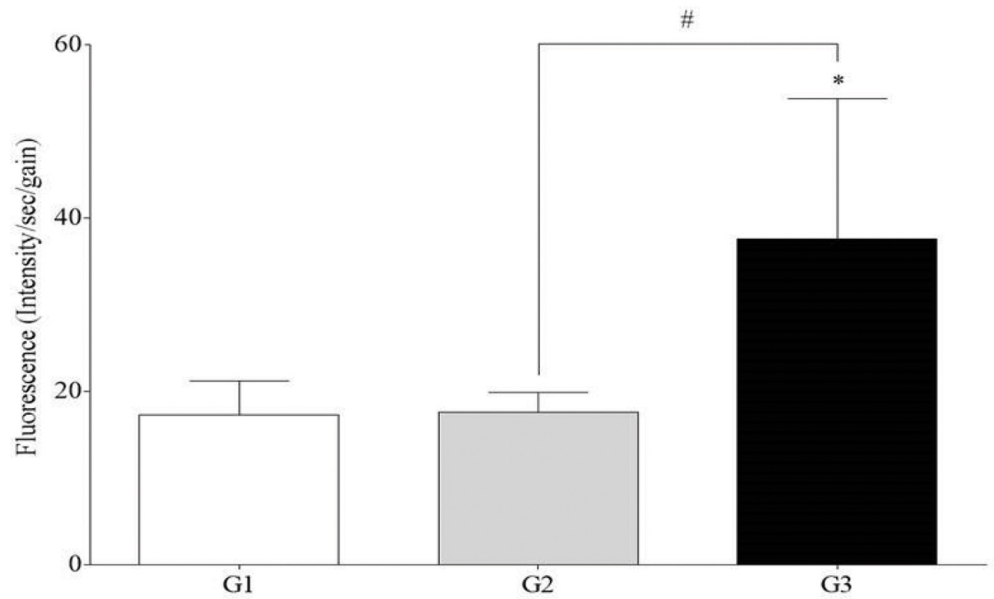

Fig. 2. (A) Fluorescence imaging of liver of mice detected by FOBI imaging system. From left to right G1 G3; (B) Quantitative analysis of liver fluorescence intensity (ROI). Note the significant increased of fluorescence intensity in liver of $\mathrm{G} 3$ compared to those of G1 or G2. "Significantly different from G1 $(p<0.05)$; "Significantly different from G2 $(p<0.05)$; Values represents shown as mean \pm standard deviation. 

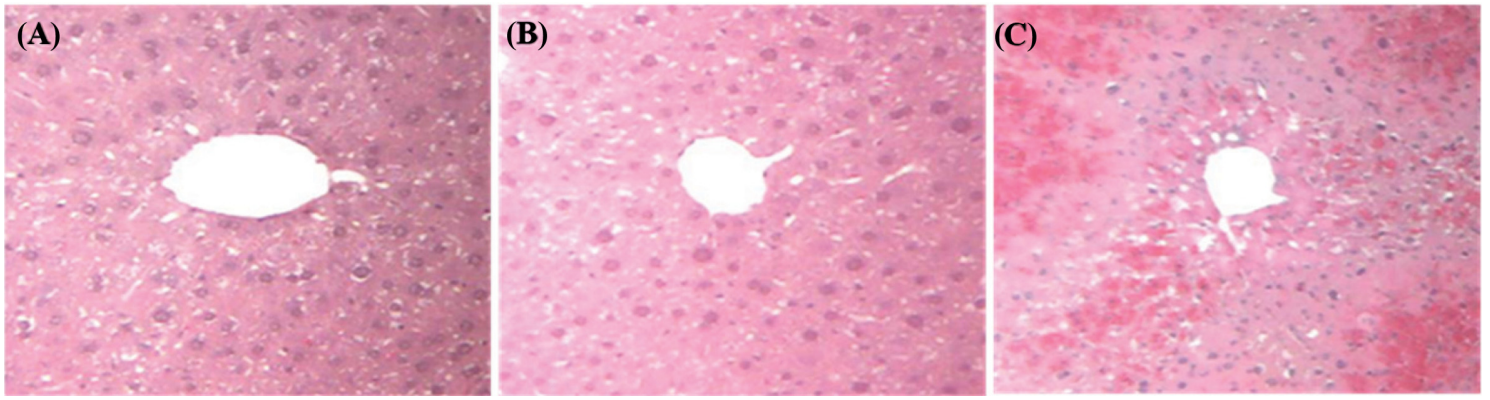

Fig. 3. Histopathological examination of liver of mice. (A) G1 as control; (B) G2 as probe-treated; (C) G3 as probe and thioacetamide (TAA) treated. Note the normal microscopic structure of liver of G1 and G2. However, in G3, numerous cell deaths are observed in mice treated with TAA. Hematoxylin \& eosin staining of paraffin embedded sections from the liver of mice. Magnification, $\times 200$.
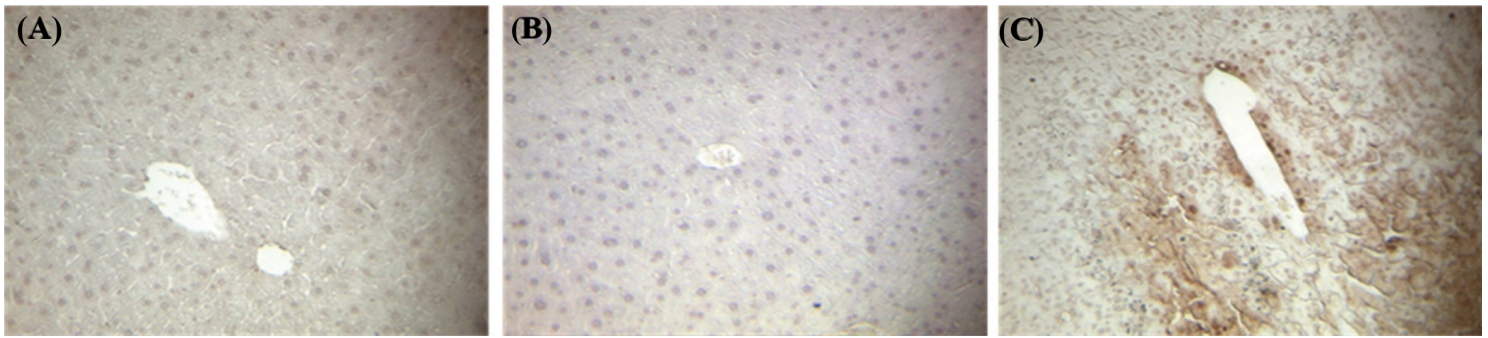

Fig. 4. Immunohistochemical examination of cell death in liver of mice. (A) G1 as control; (B) G2 as probe-treated; (C) G3 as probe and thioacetamide (TAA) treated. Note the normal microscopic structure of liver of G1 and G2. However, in G3, numerous cell deaths labeled as brown color are observed in mice treated with TAA. Immunostaining of cell death staining of paraffin embedded sections from the liver of mice. Magnification, $\times 200$.

in G1 or G2 mice (Fig. 2). Generally, the inner part of the liver showed higher fluorescent activity of Annexin-Vivo 750 than that of the surrounding liver tissues. Using analytical software, quantitative analysis of the liver fluorescence intensity (region of interest; ROI) showed that it was significantly increased in the livers of G3 mice compared with those in the livers of G1 or G2 mice $(p<0.05)$; there was no difference in the intensity level between G1 and $\mathrm{G} 2$ mice.

Histopathological examination. Histopathological examination of the liver showed that no cell death in G1 and G2 mice. However, TAA treatment induced acute hepatic damage in G3 mice (Fig. 3). Increased cell death was observed, mainly located around the central veins. In addition, disruption of hepatic cell cord and infiltration of inflammatory cells were observed.

TUNEL staining of cell death. TUNEL staining confirmed cell death features frequently in liver of G3 mice, while no cell death features were evident in livers of G1 and G2 mice (Fig. 4). Increased cell death was observed in the livers of G3 mice, especially in the centrilobular area of the liver.

\section{DISCUSSION}

For in vivo visualization of cell death, fluorescence bioimaging was used in this study to provide information about the target molecule by assessing cell death in the liver of mice, and these results generally agreed with the histopathology assessment of the liver.

To monitor disease progression, it is necessary to visualize the real-time presentation of pathological lesions in experimental animals. In this study, FMT in vivo bioimaging showed that the fluorescence intensity was increased in the right upper part of G3 mice compared with that of G2 mice. However, some limitations existed in its ability to detect the anatomical location of the liver. Although FMT has been used to image subcutaneous and superficial tissues (10) and may provide quantitative readouts from lesions (19), imaging deeply located tissues in the abdominal organs is challenging because of limited tissue penetration and light scattering, resulting in the lack of clear anatomical location. For accurate quantification, the modality should provide clear classification of the lesion and compare it with the intensity data of the fluorescence probe control.

In this study, in vivo quantification of cell death in ROI 
was attempted but failed. Because significant breakdown of the tumor vasculature results in the failure of annexinbased cell death assessment in vivo (20), high destruction of hepatic structures by severe hepatic damage may have interfered with liver imaging. However, fluorescence ex vivo bioimaging clearly showed that the fluorescence intensity was significantly increased in the livers of G3 mice compared with that in G1 and G2 mice. Additionally it represented the relatively low level of background scattering in this study compared to in vivo imaging.

Annexin-Vivo 750 was used for the detection of cell death in this study. It is a targeted fluorescence imaging agent comprising a selective protein, annexin V and a NIR fluorochrome. Annexin V interacts with the phosphatidylserine-containing phospholipid membrane and has been developed as a molecular imaging agent to measure cell death (3). As the fluorescence intensity decreased over time, estimation was carried out within $4 \mathrm{hr}$ after animal sacrifice. Because necrotic cells were more slowly removed than apoptotic cells (21) and most of the damaged cells showed a necrotic pattern in histopathological examination, it seemed that fluorescent imaging mainly detected necrotic cells. However, it was impossible to differentiate the cell death pattern in this study. Since specific fluorescence probes targeting cell death have been developed such as the phosphatidylserine-recognizing peptide (22) and histone H1-targeting peptide probe (11), further studies will be warranted to detect early dying cells during hepatic disease progression.

In HE examination, normal structures of the liver were observed in G1 and G2 mice. However, G3 mice showed severe hepatic damage. Especially, centrilobular cells were more severely damaged than periportal cells in the livers of G3 mice. TAA is thought to be mainly bioactivated by cytochrome P450 (CYP) to sulfoxide and sulfone metabolites (23). Because CYP2E1 induction is associated with elevated TAA-induced hepatotoxicity (24) and knockout of CYP2E1 reduced TAA-induced hepatotoxicity (14), CYP2E1 is a central and critical enzyme in TAA-induced toxicities and it was assumed that centrilobular cells contained several-fold higher levels of CYP2E1 than periportal cells. Although HE examination clearly showed the specific location of lesions in the liver, it was difficult to differentiate histological location using ex vivo fluorescent bioimaging in this study. Because there may be some limitations to current methods for the identification of histological location, the development of new techniques is necessary (25). A recent report showed that the application of cryosection labeling and intravital microscopy could create standard marks in the tissue of living animals (26). Further studies will be warranted to test the possibility of fluorescence examination of hepatic lesions with real histological location or to monitor biological alteration without fluorescence dye(s). Practically, three- dimensional visualization of lesions without dye-labeling could be one of these approaches (27).

Taken together, in this study, fluorescence bioimaging showed the detection of cell death and made it possible to quantify the level of cell death in male mice. The outcome was correlated with conventional biomedical examination. As it was difficult to differentiate histological location by fluorescent bioimaging, it is necessary to develop specific fluorescent dyes for monitoring hepatic disease progression and to exploit new bioimaging techniques without dye-labeling.

\section{ACKNOWLEDGMENTS}

I would like to thank Drs. Kwangmeyung Kim and Hong Yeol Yoon (Center for Theragnosis, Biomedical Research Institute, Korea Institute of Science and Technology) for their help of fluorescent bioimaging and Dr. Won Kil Lee and Ms. Joo Hye Sim for their technical assistance (Namseoul University) for their technical assistance.

Received October 10, 2017; Revised December 12, 2017; Accepted December 13, 2017

\section{REFERENCES}

1. Okada, H. and Mak, T.W. (2004) Pathways of apoptotic and non-apoptotic death in tumour cells. Nat. Rev. Cancer, $\mathbf{4}$, 592-603.

2. Krieser, R.J. and White, K. (2002) Engulfment mechanism of apoptotic cells. Curr. Opin. Cell Biol., 14, 734-738.

3. Schutters, K. and Reutelingsperger, C. (2010) Phosphatidylserine targeting for diagnosis and treatment of human diseases. Apoptosis, 15, 1072-1082.

4. Goldsworthy, T.L., Fransson-Steen, R. and Maronpot, R.R. (1996) Importance of and approaches to quantification of hepatocyte apoptosis. Toxicol. Pathol., 24, 24-35.

5. Ying, X. and Monticello, T.M. (2006) Modern imaging technologies in toxicologic pathology: an overview. Toxicol. Pathol., 34, 815-826.

6. Chen, Y., Liang, C.P., Liu, Y., Fischer, A.H., Parwani, A.V. and Pantanowitz, L. (2012) Review of advanced imaging techniques. J. Pathol. Inform., 3, 22.

7. Huh, Y.M., Jun, Y.W., Song, H.T., Kim, S., Choi, J.S., Lee, J.H., Yoon, S., Kim, K.S., Shin, J.S., Suh, J.S. and Cheon, J. (2005) In vivo magnetic resonance detection of cancer by using multifunctional magnetic nanocrystals. $\mathrm{J}$. Am. Chem. Soc., 127, 12387-12391.

8. Provencher, S.W. (2001) Automatic quantitation of localized in vivo $1 \mathrm{H}$ spectra with LCModel. NMR Biomed., 14, 260-264.

9. Weissleder, R., Tung, C.H., Mahmood, U. and Bogdanov, A., Jr. (1999) In vivo imaging of tumors with protease-activated near-infrared fluorescent probes. Nat. Biotechnol., 17, 375-378. 
10. von Wallbrunn, A., Holtke, C., Zuhlsdorf, M., Heindel, W., Schafers, M. and Bremer, C. (2007) In vivo imaging of integrin alpha $\mathrm{v}$ beta 3 expression using fluorescence-mediated tomography. Eur. J. Nucl. Med. Mol. Imaging, 34, 745-754.

11. Jung, H.K., Wang, K., Jung, M.K., Kim, I.S. and Lee, B.H. (2014) In vivo near-infrared fluorescence imaging of apoptosis using histone H1-targeting peptide probe after anti-cancer treatment with cisplatin and cetuximab for early decision on tumor response. PLOS ONE, 9, e100341.

12. Shuhendler, A.J., Pu, K., Cui, L., Uetrecht, J.P. and Rao, J. (2014) Real-time imaging of oxidative and nitrosative stress in the liver of live animals for drug-toxicity testing. Nat. Biotechnol., 32, 373-380.

13. Porter, W.R., Gudzinowicz, M.J. and Neal, R.A. (1979) Thioacetamide-induced hepatic necrosis. II. Pharmacokinetics of thioacetamide and thioacetamide- $S$-oxide in the rat. $J$. Pharmacol. Exp. Ther., 208, 386-391.

14. Kang, J.S., Wanibuchi, H., Morimura, K., Wongpoomchai, R., Chusiri, Y., Gonzalez, F.J. and Fukushima, S. (2008) Role of CYP2E1 in thioacetamide-induced mouse hepatotoxicity. Toxicol. Appl. Pharmacol., 228, 295-300.

15. Muller, A., Machnik, F., Zimmermann, T. and Schubert, H. (1988) Thioacetamide-induced cirrhosis-like liver lesions in rats--usefulness and reliability of this animal model. Exp. Pathol., 34, 229-236.

16. Dashti, H., Jeppsson, B., Hagerstrand, I., Hultberg, B., Srinivas, U., Abdulla, M. and Bengmark, S. (1989) Thioacetamide- and carbon tetrachloride-induced liver cirrhosis. Eur. Surg. Res., 21, 83-91.

17. Dasgupta, A., Chatterjee, R. and Chowdhury, J.R. (1981) Thioacetamide-induced hepatocarcinoma in rat. Oncology, 38, 249-253.

18. Kang, J.S., Morimura, K., Salim, E.I., Wanibuchi, H., Yamaguchi, S. and Fukushima, S. (2005) Persistence of liver cirrhosis in association with proliferation of nonparenchymal cells and altered location of alpha-smooth muscle actin-positive cells. Toxicol. Pathol., 33, 329-335.

19. Peterson, J.D., Labranche, T.P., Vasquez, K.O., Kossodo, S., Melton, M., Rader, R., Listello, J.T., Abrams, M.A. and Misko, T.P. (2010) Optical tomographic imaging discrimi- nates between disease-modifying anti-rheumatic drug (DMARD) and non-DMARD efficacy in collagen antibodyinduced arthritis. Arthritis Res. Ther., 12, R105.

20. Lederle, W., Arns, S., Rix, A., Gremse, F., Doleschel, D., Schmaljohann, J., Mottaghy, F.M., Kiessling, F. and Palmowski, M. (2011) Failure of annexin-based apoptosis imaging in the assessment of antiangiogenic therapy effects. EJNMMI Res., 1, 26.

21. Levin, S., Bucci, T.J., Cohen, S.M., Fix, A.S., Hardisty, J.F., LeGrand, E.K., Maronpot, R.R. and Trump, B.F. (1999) The nomenclature of cell death: recommendations of an ad hoc Committee of the Society of Toxicologic Pathologists. Toxicol. Pathol., 27, 484-490.

22. Kim, S., Bae, S.M., Seo, J., Cha, K., Piao, M., Kim, S.J., Son, H.N., Park, R.W., Lee, B.H. and Kim, I.S. (2015) Advantages of the phosphatidylserine-recognizing peptide PSP1 for molecular imaging of tumor apoptosis compared with annexin V. PLOS ONE, 10, e 0121171.

23. Hunter, A.L., Holscher, M.A. and Neal, R.A. (1977) Thioacetamide-induced hepatic necrosis. I. Involvement of the mixed-function oxidase enzyme system. J. Pharmacol. Exp. Ther., 200, 439-448.

24. Wang, X., Lu, Y. and Cederbaum, A.I. (2005) Induction of cytochrome P450 2E1 increases hepatotoxicity caused by Fas agonistic Jo2 antibody in mice. Hepatology, 42, 400410.

25. Cernohorsky, P., Kok, A.C., Bruin, D.M., Brandt, M.J., Faber, D.J., Tuijthof, G.J., Kerkhoffs, G.M., Strackee, S.D. and van Leeuwen, T.G. (2015) Comparison of optical coherence tomography and histopathology in quantitative assessment of goat talus articular cartilage. Acta Orthop., 86, 257263.

26. Ritsma, L., Vrisekoop, N. and van Rheenen, J. (2013) In vivo imaging and histochemistry are combined in the cryosection labelling and intravital microscopy technique. Nat. Commun., 4, 2366.

27. Jain, M., Robinson, B.D., Salamoon, B., Thouvenin, O., Boccara, C. and Mukherjee, S. (2015) Rapid evaluation of fresh ex vivo kidney tissue with full-field optical coherence tomography. J. Pathol. Inform., 6, 53. 